\title{
Spin- and Angle-Resolved Spectroscopy and Spin Waves in Biogenic Magnonic Systems.
}

\begin{abstract}
This review paper covers aspects of spin waves excitation and propagation as magnons (a quasiparticle of excited spin waves analogous to phonons and plasmons which are also quasiparticles of quanta of vibrational waves and electromagnetic waves propagating along a metal surface respectively) how they can be biologically engineered for applications and probing the biogenic magnonic nanoparticle using lab-scale spin and angular resolved photoemission spectroscopy to obtain more details on spin waves.
\end{abstract}




\subsection{Short Review of Photoemission Spectroscopy}

Photoemission spectroscopy is based on the simple principle of photoelectric effect where electrons are ejected from material surfaces using high energy radiation. The emitted electron's momentum (at angle relative to the material surface's normal) and energy are related to that of the electron in the photoelectric material. The emitted electron's intensity for a given range of energies and angular distributions is being measured in an ARPES experiment, broken down into spectral intensity measured as a function of the electron's momentum and is demonstrated using an Energy Distribution Curve (EDC).

Light sources used in photoemission spectroscopy are usually synchrotron light source or solid-state lasers. Synchrotron light beam is produced by the acceleration of energetic particles like electrons about a constant magnetic field in circular path, the resulting radiation (about $20-200 \mathrm{eV}$ ) is polarized and used in beamline for experiments. Lasers are capable of producing photons of energies 5-7 eV (which exceeds work function of most materials), their lower energy level allows a much higher energy resolution - helping to reduce background noise.

Detector helps to measure the state of electrons near the surface of materials, before entering the detector, a retarding potential is applied to lower the energy and improve the energy resolution. Upon entering the detector, the electrons are first collimated using a series of electrostatic lenses (to increase momentum spread), then a half sphere electron deflector is used to select the energy of the photoelectrons. [12]

\subsection{Spin and Angular Resolved Photoemission Spectroscopy (SARPES)}

Spin and Angular Resolved Photoemission Spectroscopy is a great tool in studying exotic materials with spin-polarized surface states like topological insulators. Angular resolved photoemission spectroscopy (ARPES) helps study the band structure of electronic materials by measuring the angle at which photoelectrons are emitted from a material (a measure proportional to the crystal momentum) when illuminated by light from high energy sources like synchrotron. To determine if the bands have spin-degeneracy (i.e. containing 
electrons with spin states allowing equal energy level), the material is probed by polarizing light from high energy sources. Spin splitting of valence energy bands by spin-orbit coupling in materials without inversion symmetry can be noticed by Spin-resolved ARPES (SARPES). The SARPES utilizes the spin-dependence in scattering experiments to characterize the spin-polarization (a function of spectral intensity) of electrons with momentum using a spectrometer to measure spectra corresponding to different spin axis.

F. Bussolotti et al (2016) described SARPES set-up used to study quantum phenomena in low dimensional electronic systems. The set up has an energy (angular) resolution of $<12 \mathrm{meV}\left(0.2^{\circ}\right)$, the high energy and angular resolution of the ARPES system combined with spin detection capability and accessible photon energy \& temperature range makes it unique to study quantum phenomena in low dimensional electronic systems, it also allows upgrade of anxilliary systems (for studying organic systems) and photon sources. [2] The paper demonstrated SARPES abilities using Gold [Au(111)] and Molybdenum disulphide $\left(\mathrm{MoS}_{2}\right)$.

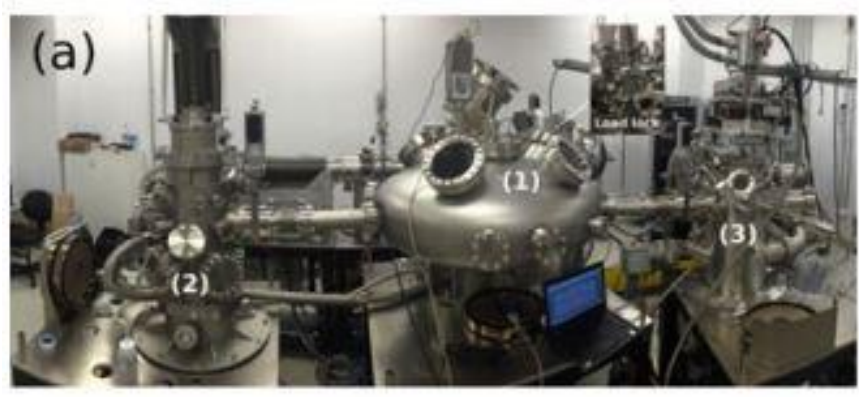

(b)

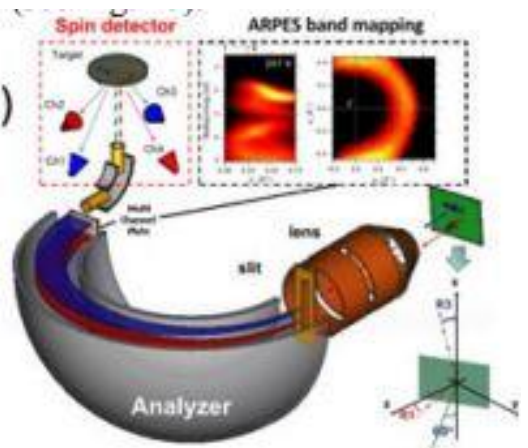

Figure 1. Picture of SARPES set-up described in F. Bussolotti et al (2016).

Reprinted with permission from [2]. (C) 2016 Materials Research Society/Springer Nature

The ability of the setup to allow ancillary systems for organic matter and the spin detection capacity makes it a good equipment for studying spin waves propagating from the surface of a biogenic nanoparticles. 


\subsection{Introduction to Magnonics}

Magnonics is a field involving the propagation of spin waves in magnetic materials. Magnons, which are a quanta of excitations from systems with strong magnetic ordering, similar to Phonons and Plasmons which are also quasiparticles of excitations in condensed matter, have promising capabilities for future information processing.

Spin waves which arise from precession of magnetic moments are a propagating re-ordering of the magnetisation in a material. They can propagate in magnetic media with magnetic ordering (ferromagnets and antiferromagnets). The precession frequency of spin waves range from $1-100 \mathrm{GHz}$ depending on the magnetic material used and can go up to the $\mathrm{THz}$ range because of resonance.

Magnon-magnon scattering, magnon-phonon scattering and loss by eddy current leads to damping of propagating spin waves. In magnonic systems, the Landau-Lifshitz equation describes magnetization dynamics. They have the general form

$$
\frac{\partial M(r, t)}{\partial t}=-\gamma \mu_{0} \mathbf{M}(\mathbf{r}, \mathrm{t}) \times \mathbf{H}_{\mathrm{eff}}(\mathbf{r}, \mathrm{t})
$$

Where $\mathbf{M}$ and $\mathbf{H}_{\text {eff }}$ are the magnetization and effective magnetic field respectively, $\gamma$ represents the gyromagnetic ratio.

Krivoruchko and Savchenko, 2018 explored the use of electric field to control the power flow of spin waves in thin ferromagnetic films by strengthening spinorbit in ions present in the material, causing a frequency shift of spin which is evaluated using an isofrequency curve. This result could help in creating magnonic devices which can be tuned electrically [11].

In [14], broadband spectroscopy was used to detect magnons excited electrically. [15] [16] reported that magnetoresistance arise from propagation of spin waves. Resistance of metal deposited at a magnon junction is dependent on the magnetization of the layers at the magnon junction in [15].

\subsection{Hartman Effect}

This is a phenomenon that describes the tunnelling of wave packets through a potential barrier in metal-insulator-metal connections. Hartman noticed that the time taken for wave packets to travel from one point of a potential barrier to another (group delay) is less than that which the packet requires to travel the same distance without a 
barrier in a material i.e. the lengthening of barrier width causes saturation of group delay.

The Hartman effect was demonstrated in [12], where exchange spin waves of $\mathrm{GHz}$ frequency tunnelling through a barrier in a thin film of perpendicular magnetic anisotropy showed saturation for group delay after expanding the thickness of the barrier - this was calculated using the transmissivity of the spin waves (which the group delay depends on)

$$
\tau_{\mathrm{gr}}(\Omega, L)=\frac{1}{\gamma \mu_{o} H_{o}} \frac{d}{d \Omega}\left[\operatorname{Arg}(T)+k_{\mathrm{m}} \mathrm{L}\right]
$$

where $\tau_{\mathrm{gr}}$ is the group delay, $\mathrm{T}$ is the transmissivity, $\mathrm{L}$ is the width of the barrier and $k_{\mathrm{m}} \mathrm{L}$ is the geometrical phase.

\subsection{Magnonic Dispersion Relation}

The dispersion relation, where energy is plotted alongside k-space, can be determined experimentally by Brillouin scattering [18]. The momentum of spin waves is demonstrated using reciprocal wave vector $\boldsymbol{k}$, and the energy which is a function of wave number gives the dispersion relation for a 1 dimensional ferromagnet:

$$
\hbar \omega=\frac{4 J S}{\hbar}[1-\cos (k a)]
$$

Where $\omega$ is the angular frequency o spin waves, $S$ is electron spin (assumed as $\frac{\pi}{2}$ ), $J$ is the exchange energy and $a$ is the distance between spins.

This change for a cubic lattice in 3 dimensions to:

$$
\hbar \omega=\frac{2 J S}{\hbar}\left[\mathrm{z}-\sum_{\delta} \cos (k . \delta)\right]
$$

For 1 dimensional dispersion relation, if $(k a) \ll 1$, we have a parabolic dispersion:

$$
\hbar \omega \approx 4 J S a^{2} k^{2}
$$

\subsection{Ferromagnetic Resonance Spectra}

In ferromagnetic resonance, EM microwaves excite electron spin in magnetically ordered materials which are present in a non-dynamic magnetic field. Precession of magnetic moments occurs in the materials when an applied magnetic field causes resonance (when the 
microwave frequency is equivalent to the precession frequency given by the Larmor frequency

$$
\omega_{\mathrm{L}}=\gamma \mu_{\mathrm{o}} \mathrm{H}
$$

where $\gamma$ is the gyromagnetic ratio $\mu_{0}$ is the magnetic permeability and $\mathrm{H}$ is the magnetic field.

This leads to absorption of energy that causes the spectrum to reach its peak position. The width of the spectrum depicts damping of precession and spin lattice relaxation.

Ferromagnetic resonance peak can also be demonstrated in terms of the gyromagnetic ratio $\gamma$ and magnetic anisotropy. A Lorentzian function of the peak is given by:

$$
f(H)=I\left[\frac{\left(\frac{\Delta H_{\omega}}{2}\right)^{2}}{\left(H-H_{O}\right)^{2}+\left(\frac{\Delta H_{\omega}}{2}\right)^{2}}\right]
$$

Where $\mathrm{I}$ is the peak signal, $\mathrm{H}_{\mathrm{o}}$ is the peak position and $\Delta \mathrm{H}_{\omega}$ is the full width at half maximum.

\subsection{AntiFerromagnetic Magnons}

The magnon dispersion relation for antiferromagnets with two types of anisotropy: easy-axis $\left(\mathrm{MnF}_{2}\right.$ and $\left.\mathrm{FeF}_{2}\right)$ and easy-plane (NiO) was discussed in [20].

Magnons transport spin currents (flow of spin angular momentum) in ferromagnetic materials. The density of spin current with polarization $z$ which is propagated by spin waves of wave vector $k$ and energy $\varepsilon_{k}$ is given by:

$$
J_{s}^{Z}=\frac{\hbar}{(2 \pi)^{3}} \int d^{3} k v_{k}\left[n_{k}(r)-n_{k}^{o}\right]
$$

Where $v_{k}$ is velocity of spin waves, $n_{k}(r)$ is the number of magnons with wave vector $k$ at position $r$ and $n_{k}^{o}$ is the number in thermal equilibrium [20].

For antiferromagnets spin current propagates through 2 magnon modes so the magnon spin current density is given as:

$$
J_{s}^{Z}=-\frac{\hbar}{(2 \pi)^{3}} \int d^{3} k\left[v_{a k}\left(n_{a k}(r)-n_{a k}^{o}\right)-v_{\beta k}\left(n_{\beta k}(r)-n_{\beta k}^{o}\right)\right]
$$


The paper also discussed excitation of spin waves in antiferromagnets using spin Seebeck effect where thermal gradients create magnonic spin currents.

\subsection{Spin Phenomena in Magnetic Bioparticles}

Magnetosome chains in magnetotactic bacteria mimics magnetic nanoparticle chains, a magnetosome chain is equivalent to about 10 to 20 magnetite particles where the dipolar moments sum up to create a fixed intracellular compass needle that allows the bacteria's cell body to be aligned with the Earth's magnetic field [21].

The particles in a magnetosome chain only interact magnetically by dipolar coupling; they tend to show exciting spin-wave dynamics.

Zingsem et al, 2019 described the use of biogenic nanoparticle chains of bacteria Magnetospirillium gryphiswaldense (strain MSR-1, wildtype) to nanoscale magnonics at room-temperature [3]. The ferromagnetic resonance spectra (FMR) of the magnetosome chain shows magnonic band gaps and also that geometry of magnetosome chain, which depends on the genotype of the bacteria, determines band structure (confirming their magnonic nature). 

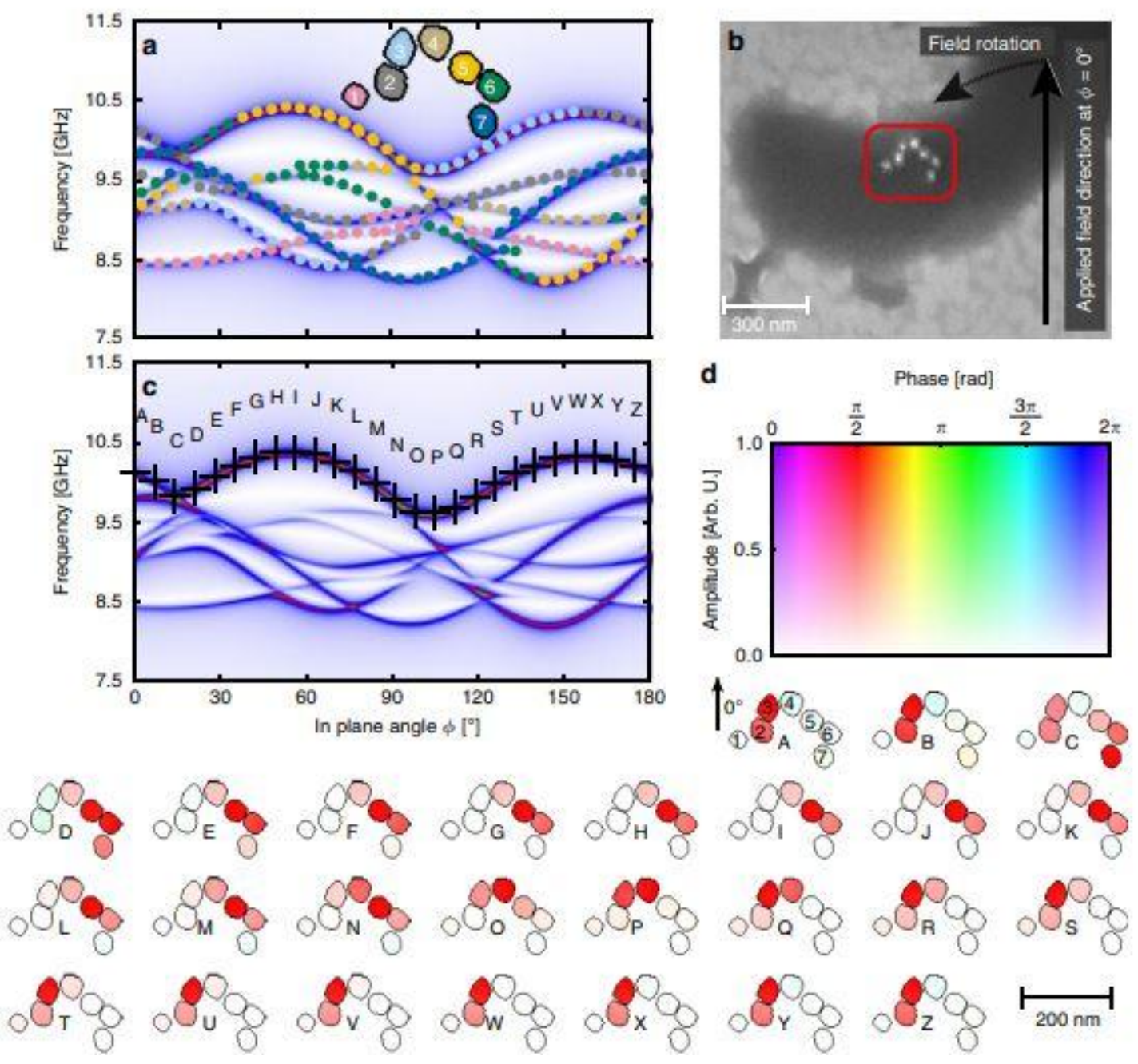

Figure 2, Correlation of magnonic fine structure and geometric configuration of particles. A) Simulated FMR spectra of seven magnetic nanocrystals in a coiled arrangement reproduced as silhouettes from an electron micrograph $B$ ) of a cell of the AmamK-mutant of MSR-1 C) Analysis of the spatial contributions to the upperenvelope line of the spectrum. Each letter corresponds to a position on the envelope and the phase and amplitude of the magnonic response is mapped according to the color scale D) onto each point of the simulation grid (2.6 $\mathrm{nm}$ mesh size). Phase and amplitude are uniform within a given particle, with red hue indicating resonant response $\left(90^{\circ}\right.$ phase relative to excitation) and cyan hue indicating opposite-toresonant response. Reprinted with permission from [3]. Copyright (C) 2019 Benjamin W. Zingsem et al 


\subsection{Biogenic Magnonic Particle Study With SARPES}

While FMR and MFM might reveal magnonic and magnetic properties of these biogenic particles, a spin- and angular resolved photoemission spectroscopy of these biogenic nanoparticles might be used to probe spinpolarization along the surface of the magnetosome chains.

The SARPES set up could be upgraded to allow attachment of ancilliary systems (for studying organic systems) and laser source which makes it apt as an additional technique for probing biogenic magnonic nanoparticles excited by laser pulses.

SARPES could reveal details such renomalization of bosonic quasiparticle (like magnons) band dispersions because of the interacting modes. Single particle spectral function can be obtained in terms of electron momentum and energy. Dispersion renormalization is obtained as abrupt drifts in slopes on an electronic dispersion curve [25].

Room-temperature magnonics from biogenic molecules could be used in MRI and NMR for spin relaxation effects. Sloan, 2019 reported using surface magnon polaritons in the negative magnetic permeability materials to strongly enhance spin relaxation in the nearby emitters (spin transition in the THz range) [28].

In Nuclear Magnetic Resonance (NMR), excited magnetic state returns to equilibrium distribution by relaxation, there is the possibility for using surface magnon polariton from genetically engineered magnonics from biogenic particles to enhance relaxation in biological systems in Nuclear Magnetic Resonance and Magnetic Resonance Imaging. 


\subsection{Periodicity in Magnetic Metamaterials}

Periodicity in magnetic metamaterials helps control the propagation of spin waves and the energy at the nanoscale. In magnetic materials, properties arise from structuring rather than intrinsic properties. Patterning of periodic structures causes exciting properties to emerge in these materials. For example, degenerate states split allowing no magnetic modes in the magnonic band gap. Changing the periodicity by altering the size of the components in theory gives the flexibility to manipulate selective propagation of spin waves frequencies filtering out some magnonic modes.

Magnonic bandgaps form when dipoles of adjacent particles in a magnonic structure are coupled. The coupling is determined by the size of the constituent particles and their separation distance, large particles with small separation distance would have a larger magnonic bandgap. An FMR spectra analysis of magnetosome chains of magetotactic bacteria show a $180^{\circ}$ periodicity in their geometry, and dashed lines reveal the prevailing uniaxial shape anisotropy of the two linear chain segments, as confirmed with a micromagnetic model [3].

Magnonic Crystals, magnetic metamaterials that have alternating magnetic properties due to small spatial inhomogeneities that creates macroscopic behaviour, share similarities with Biogenic Magnonic molecules since they both derive their magnonic properties through geometric structuring of their constituent magnetic particles. Tuning of the size of bandgaps in magnonic crystals leads to selective propagation of spin wave frequencies and is achieved by alternating parameters such as the relative permeability or saturation magnetization. 


\subsection{Conclusion}

The possibility of achieving room temperature spin wave transport using genetically modified sustainable bioparticles promises exciting applications not just because of the environmental footprint but also the use in information processing devices that require low power and dissipate less heat.

Lab-scale Spin and Angular Resolved Photoemission Spectroscopy (SARPES) could be added to a list of experimental techniques for probing biogenic magnonic nanoparticles light.

The limitation of the work is that so much work has not been carried out on exciting spin waves in biogenic nanoparticles using pulsed femtosecond lasers as opposed to exciting magnetism in materials using this type of pulsed lasers. Gotlieb et al, 2013 demonstrated the use of pulsed lasers attached to SARPES setup for study of L-gap $\mathrm{Au}(111)$ surface states [26].

\section{Acknowledgement}

I acknowledge Springer Nature and the Materials Research Society for the license given to reuse figure1. 


\subsection{References}

[1]L. Wang, L. Gao, L. Jin , Y. Liao, T. Wen, X. Tang, H. Zhang, and Z. Zhong, Magnonic waveguide based on exchange-spring magnetic structure, AIP Advances $\quad \mathbf{8}, 055103 \quad$ (2018); https://doi.org/10.1063/1.5018637.

[2] Bussolotti, F., Zhang, Z., Kawai, H., \& Goh, K. (2017). A Lab-scale Spin and Angular Resolved Photoemission Spectroscopy Capability for 2D Valleytronics. MRS Advances, 2(29), 1527-1532. doi:10.1557/adv.2016.626

[3] B. W. Zingsem, T. Feggeler, A. Terwey, S. Ghaisari, D. Spoddig, D. Faivre, R. Meckenstock, M. Farle and M. Winklhofer, Biologically encoded magnonics, NATURE COMMUNICATIONS (2019) 10:4345 | https://doi.org/10.1038/s41467-019-12219-0

[4] S. Kosen, R. G. E. Morris, A. F. van Loo, A. D. Karenowska, Measurement of a magnonic crystal at millikelvin temperatures, Appl. Phys. Lett. 112, 012402 (2018); https://doi.org/10.1063/1.5011767

[5] A. F. van Loo, R. G. E. Morris, A. Karenowska, Time-resolved measurements of surface spin-wave pulses at millikelvin temperatures, Phys. Rev. Applied 10, $044070 \quad$ (2018); https://doi.org/10.1103/PhysRevApplied.10.044070

[6] B. Lenk, H. Ulrichs, F. Garbs, M. Münzenberg, The building blocks of magnonics, Physics Reports, vol. 507, issues 4-5, 2011; https://doi.org/10.1016/j.physrep.2011.06.003

[7] M. Muntwiler, M. Hoesch, V. N. Petrov, M. Hengsberger, L. Patthey, M. Shi, M. Falub, T. Greber, J. Osterwalder, Spin- and angle-resolved photoemission spectroscopy study of the Au(111) Shockley surface state, Journal of Electron Spectroscopy and Related Phenomena, volumes 137-140, 2004, Pages 119-123, ISSN 0368-2048, https://doi.org/10.1016/j.elspec.2004.02.048

[8] K. Kuroda, K. Yaji, A. Harasawa, R. Noguchi, T. Kondo, F. Komori, S. Shin, Experimental Methods for Spin- and Angle- Resolved Photoemission Spectroscopy Combined with Polarization-Variable Laser, Jove (2018); doi: 10.3791/57090

[9] M. Fanciulli, J. Schusser, M. Lee, Z. El Youbi, O. Heckmann, M. C. Richter, C. Cacho, C. Spezzani, D. Bresteau,J-F Hergott, P. 
D’Oliveira, O. Tcherbakoff, T. Ruchon, J. Minar, K. Hricovini; Spin- , time- and angle-resolved photoemission spectroscopy on $\mathrm{WTe}_{2}$; Phys. Rev. Research 2, $013261 \quad$ (2020); https://doi.org/10.1103/PhysRevResearch.2.013261

[10] A. Damascelli, Probing the Electronic Structure of Complex Systems by ARPES, Physica Scripta, 61 (2004)

[11] V. N. Krivoruchko, A. S. Savchenko, Electric Field Control of Magnon Power Flow in Thin Ferromagnet Films, Acta Physics Polonica A 3, vol. 133 (2018), doi: 10.12693/APhysPolA.133.463

[12] J. W. Kłos, Y. S. Dadoenkova, J. Rychły, N. N. Dadoenkova, I. L. Lyubchanski, J. Bamas, Hartman effect for spin waves in exchange regime, Scientific Reports, (2018)8: 17944, doi: 10.1038/s41598-01835761-1

[13] Golovchan A.V., Kruglyak W., Tkachenko V.S., Kuchko A. N. 2018. Magnonic band spectrum of spin waves in an elliptical helix. $R$. Soc. open sci.5:172285. http://dx.doi.org/10.1098/rsos.172285

[14] Che, P., Baumgaertl, K., Kúkol'ová, A et al. Efficient wavelength conversion of exchange magnons below $100 \mathrm{~nm}$ by magnetic coplanar waveguides. Nat Commun 11, 1445 (2020). https://doi.org/10.1038/s41467-020-15265-1

[15] Guo, C.Y., Wan, C.H., He, W.Q. et al. A nonlocal spin Hall magnetoresistance in a platinum layer deposited on a magnon junction. Nat Electron 3, 304-308 (2020). https://doi.org/10.1038/s41928-020$\underline{0425-9}$

[16] V. D. Nguyen, C. Naylor, L. Vila, A. Marty, P. Laczkowski, C. Beigne, L. Notin, Z. Ishaque, J.P. Attané, Magnon magnetoresistance of NiFe nanowires: size dependence and domain wall detection, Appl. Phys. Lett. 99, 262504 (2011); https://doi.org/10.1063/1.3672828

[17] F. Groß, M. Zelent, N. Träger, J. Förster, U.T. Santi, R. Sauter, M. Deeker, C.H. Back, M. Weigand, K. Keskinbora, G. Schütz, M. Krawczyk, J. Gräfe, Building Blocks for Magnon Optics: Emission and Conversion of Short Spin Waves, ACS Nano 2020, 14, 12, 1718417193. https://doi.org/10.1021/acsnano.0c0706

[18] Håkonsen V, 2015, Self-Assembly of Magnetic Nanoparticles into Superstructures for Magnonics [Master Thesis], [NTNU Trondheim]: Norwegian University of Science and Technology. 
[19] Wu X., Liu Z., Luo T., Magnon and Phonon Dispersion, Lifetime and Thermal Conductivity of Iron from Spin-Lattice Dynamics Simulations, Journal of Applied Physics 123, 085109 (2018); https://doi.org/10.1063/1.5020611

[20] Rezende S.M., Azevedo A., Rodríguez-Suárez R.L., Introduction to antiferromagnetic magnons, J. Appl. Phys. 126, 151101 (2019); https://doi.org/10.1063/1.5109132

[21] Winklhofer M., Spin dynamics of a magnetic nanoparticle chain, MA 27: PhD Focus Session: Biogenic spin phenomena (joint session MA/AKjDPG), Regensburg 2019

[22] Staňo M., Fruchart O., Handbook of Magnetic Materials, North Holland, Elsevier, 2018

[23] Schmitt F., Kirchmann P.S., Bovensiepen U., Moore R.G., Chu J.H., Lu D.H., Rettig L., Wolf M., Fisher I.R., Z-X Shen, Ultrafast electron dynamics in the charge density wave material $\mathrm{TbTe}_{3}, \mathrm{New}$ Journal of Physics 13 (2011) 063022

[24] Nishide A., Takeichi Y., Okuda T., Taskin A.A., Hirahara T., Nakatsuji K., Komori F., Kakizaki A., Ando Y., Matsuda I., Spinpolarized surface bands of a three-dimensional topological insulator studied by high-resolution spin- and angle- resolved photoemission spectroscopy, New Journal of Physics 12 (2010) 065011. Doi: 10.1088/1367-2630/12/6/065011

[25] Bossini, D., Konishi, K., Toyoda, S. et al Femtosecond activation of magnetoelectricity. Nature Phys 14, 370-374 (2018). https://doi.org/10.1038/s41567-017-0036-1

[26] Gotlieb K., Hussain Z., Bostwick A., Lanzara A., Jozwiak C., Rapid high resolution spin- and angle- resolved photoemission spectroscopy with pulsed laser source and time-of-flight spectrometer, Review of Scientific Instruments 84, $093904 \quad$ (2013); https://doi.org/10.1063/1.4821247

[27] van Loo A.F., Morris R.G.E, Karenowska A.D., Time- Resolved Measurements of Surface Spin-wave Pulses at Milikelvin Temperatures, Phys. Rev. Applied 10, 044070. Doi: 10.1103/physrevapplied.10.044070

[28] Sloan J., Rivera W., Joannopoulos J.D., Kaminer I., Soljačić M., Controlling spins with surface magnon polaritons, Phys. Rev. B 100, 235453, 2019. https://doi.org/10.1103/PhysRevB.100.235453 
